\title{
Cohomology groups of units in $\mathbb{Z}_{p}^{d}$-extensions
}

\author{
by \\ Mingzhi Xu (Columbus, Ohio)
}

In this paper, $K$ is an arbitrary number field and $p$ is a prime number. Let $\mathbb{Z}_{p}$ be the $p$-adic integers and let $K_{\infty}$ be a Galois extension of $K$ such that $\mathcal{G}=\operatorname{Gal}\left(K_{\infty} / K\right) \cong \mathbb{Z}_{p}^{d}, d \in \mathbb{Z}, d \geq 1$. For an arbitrary field $F$ between $K$ and $K_{\infty}$, let $\mathcal{E}(F)$ be the group of global units of $F$ and let $\mathcal{E}(F)^{\text {univ }}$ be the intersection $\bigcap_{L \subset K_{\infty}, L / F \text { finite }} N_{L / F}(\mathcal{E}(L))$. The Iwasawa algebra $\mathbb{Z}_{p}[[\mathcal{G}]]$ will be denoted by $\Lambda$. An ideal in $\Lambda$ that contains two elements that are relatively prime will be called an ideal of height at least two. For a set $S$ of primes in $K$ above $p, M_{S}(F)$ denotes the maximal abelian $p$-extension of $F$ which is unramified outside of $S$, and let $X_{S}(F)=\operatorname{Gal}\left(M_{S}(F) / F\right)$.

If $F$ is finite over $K$, then $A(F)$ will be the $p$-part of the ideal class group of $F$, and for a prime $\wp \subset K, U_{\wp}(F)$ will be the group of local units of $F \otimes_{K} K_{\wp}$ which are congruent to 1 modulo the primes above $\wp$. The product $\prod_{\wp \in S} U_{\wp}(F)$ is denoted by $U(F)$. The closure of $\mathcal{E}(F) \cap U(F)$ in $U(F)$ is written as $\overline{\mathcal{E}}(F)$. If $F$ is infinite over $K$, we define $A(F), \overline{\mathcal{E}}(F)$ and $U(F)$ to be the inverse limits $\lim A(L), \lim _{\overline{\mathcal{E}}}(L)$ and $\lim _{U} U(L)$ respectively, where the inverse limits are over finite extensions $L$ of $K$ such that $L \subset F$, and are with respect to norm maps. Define $T(F)$ to be the set of primes of $K$ which ramify in $K_{\infty} / F$, and let $r_{1}$ and $r_{2}$ be the numbers of real and complex primes of $K$.

Suppose $F$ is finite over $K$, and let $r_{1}(F)$ and $r_{2}(F)$ be the numbers of real and complex primes of $F$. Then $\operatorname{rank}_{\mathbb{Z}} \mathcal{E}(F)=r_{1}(F)+r_{2}(F)-1$. Hence we must have $\overline{\mathcal{E}}(F) \cong \mathbb{Z}_{p}^{c} \times B$, where $c \leq r_{1}(F)+r_{2}(F)-1$ and $B$ is finite. Let $\delta_{F}=r_{1}(F)+r_{2}(F)-1-c$. For a general $F$, if the set $\left\{\delta_{L}: L \subset F, L / K\right.$ finite $\}$ is bounded, then we say that the weak Leopoldt hypothesis holds for $F$ and $S$.

Fix a set $S$ of primes in $K$ above $p$. If $\wp$ is any prime in $S$ and $F$ is finite over $K$, let $v$ be a prime of $F$ lying above $\wp$ and let $F_{v}^{*}$ be the multiplicative group of $F_{v}$, the completion of $F$ at $v$. Following Wintenberger ([12]), we

1991 Mathematics Subject Classification: 14H25, 14G10, 14G25. 
define $Z\left(F_{v}\right)$ to be $\lim _{n} F_{v}^{*} /\left(F_{v}^{*}\right)^{p^{n}}$. If $F / K$ is an infinite extension, $Z\left(F_{v}\right)$ is defined to be $\lim Z\left(L_{q}\right)$, where the inverse limit is over finite extensions $L$

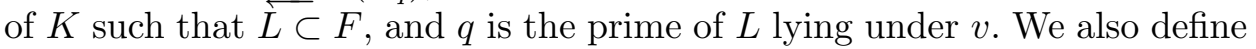
$Z_{\wp}(F)=\varliminf_{\longleftarrow} \prod_{q \mid \wp, q \subset L} Z\left(L_{q}\right)$, where the inverse limit is over finite extensions $L$ of $K$ such that $L \subset F$. Observe that for any $F$ with $K \subset F \subset K_{\infty}$, we have $U_{\wp}(F) \subset Z_{\wp}(F)$.

If $H$ is a closed subgroup of $\mathcal{G}$, define $I(H)$ to be the ideal of $\Lambda$ generated by $\{\gamma-1: \gamma \in H\}$. If $H=\operatorname{Gal}\left(K_{\infty} / F\right)$, we also write $I(H)$ as $I(F)$, and we define $\Lambda_{H}$ to be $\Lambda / I(H)=\mathbb{Z}_{p}[[\mathcal{G} / H]]$. For convenience, we let $X(F)=$ $X_{S}(F)$. The maps

$$
\begin{aligned}
& \pi_{X}: X\left(K_{\infty}\right) /\left(I(F) X\left(K_{\infty}\right)\right) \rightarrow X(F), \\
& \pi_{A}: A\left(K_{\infty}\right) /\left(I(F) A\left(K_{\infty}\right)\right) \rightarrow A(F), \\
& \pi_{U}: U\left(K_{\infty}\right) /\left(I(F) U\left(K_{\infty}\right)\right) \rightarrow U(F), \\
& \pi_{\mathcal{E}}: \overline{\mathcal{E}}\left(K_{\infty}\right) /\left(I(F) \overline{\mathcal{E}}\left(K_{\infty}\right)\right) \rightarrow \overline{\mathcal{E}}(F)
\end{aligned}
$$

will be the natural projection maps.

Before we state the main results, let us state the exact assumptions. We assume that the Iwasawa- $\mu$-conjecture is true for $K$. We also assume that for every $\mathbb{Z}_{p}$-extension $F$ of $K$ such that $F \subset K_{\infty}$, the weak Leopoldt hypothesis holds for $F$ and $S$. In addition, we assume that for any finite extension $F$ of $K$ such that $F \subset K_{\infty}$, Leopoldt's conjecture holds for $F$.

Our main result is: Let $F$ be any field between $K$ and $K_{\infty}$. For any integer $i \geq 0$, there exist a positive integer $n$ and an ideal $\mathcal{A}$ of height at least two in $\Lambda$, both independent of $F$, such that

$$
I_{T(K)}^{n} \mathcal{A} H^{i}\left(\operatorname{Gal}\left(K_{\infty} / F\right), \mathcal{E}\left(K_{\infty}\right)\right)=0 .
$$

When $d=1$, this was proved by Iwasawa ([5]). Greenberg ([3]) proved many fundamental results when $d \geq 2$ and $S$ is the set of all primes above $p$. In [9], Rubin proved a key result (Theorem 7.6(i)) for the case when $d=2$ and $K$ is an imaginary quadratic field, which will be generalized to prove our result.

In addition, the rank of $X_{S}\left(K_{\infty}\right)$ will be given by a formula which generalizes a result of Greenberg. The more general module $X_{S}(F)$ is also considered and the result can be found in Theorem 2.2.

1. The $\Lambda$-modules $U\left(K_{\infty}\right), X\left(K_{\infty}\right)$ and $A\left(K_{\infty}\right)$

Lemma 1.1. For $\wp \in S$, let $D_{\wp}$ be the decomposition group of $\wp$ in $K_{\infty} / K$. Let $\pi_{Z, \wp}$ be the natural projection: $Z_{\wp}\left(K_{\infty}\right) / I(F) Z_{\wp}\left(K_{\infty}\right) \rightarrow Z_{\wp}(F)$. Then $I\left(D_{\wp}\right)^{d-1} \operatorname{ker}\left(\pi_{Z, \wp}\right)=0$.

Proof. This follows from Lemma 5.2 in [12] and induction. 
If $Q$ is any set of primes of $K$ above $p$, then the product $\prod_{\wp \in Q} I\left(D_{\wp}\right)$ will be written as $I_{Q}$.

TheOREM 1.2. We have

$$
I_{T(F) \cap S} \operatorname{coker}\left(\pi_{U}\right)=0 \quad \text { and } \quad I_{T(F) \cap S}^{d} \operatorname{ker}\left(\pi_{U}\right)=0 .
$$

Proof. When $d=2$ and $K$ is imaginary quadratic, this was proved by Rubin in Theorem 5.1(i) of [9]. The proof here is similar. More precisely, it follows from Lemma 1.1 and class field theory by looking at $\operatorname{coker}\left(\pi_{U}\right)_{\wp}$ and $\operatorname{ker}\left(\pi_{U}\right)_{\wp}$ for each $\wp \in S$.

Lemma 1.3. Let $L_{\infty}$ be an abelian extension of $K_{\infty}$ that is Galois over $K$ and let $Z=\operatorname{Gal}\left(L_{\infty} / K_{\infty}\right)$. Suppose $L_{1}$ is the fixed field of $I(F) Z$ and $L_{2}$ is the maximal abelian extension of $F$ in $L_{\infty}$. Then $L_{2} \subset L_{1}$, and $\operatorname{Gal}\left(L_{1} / L_{2}\right)$ is finitely generated over $\mathbb{Z}_{p}$. Also $\mathcal{G}$ acts trivially on $\operatorname{Gal}\left(L_{1} / L_{2}\right)$. If $\operatorname{Gal}\left(K_{\infty} / F\right)$ is cyclic, then $L_{1}=L_{2}$.

This is exactly Lemma 5.2 of [9]. From the proof given there, we see that if $\alpha_{1}, \ldots, \alpha_{n} \in \operatorname{Gal}\left(L_{1} / F\right)$ generate $\operatorname{Gal}\left(K_{\infty} / F\right)$, then $\operatorname{Gal}\left(L_{1} / L_{2}\right)$ is generated by the commutators $\left[\alpha_{i}, \alpha_{j}\right], 1 \leq i \leq n, 1 \leq j \leq n$.

A $\Lambda_{H}$-module $M$ is called a torsion $\Lambda_{H}$-module if $M$ can be annihilated by an element $\alpha$ in $\Lambda_{H}$ which is not a zero divisor. For any $\Lambda$-module $Y$, let $Y^{H}=\{y \in Y: h y=y$ for all $h \in H\}$ and $Y_{H}=Y / I(H) Y$.

Lemma 1.4. Suppose $H \subset \mathcal{G}$ and $0 \rightarrow Y \rightarrow Z \rightarrow W \rightarrow 0$ is an exact sequence of $\Lambda$-modules. Then there is an exact sequence

$$
H_{1}(H, Z) \rightarrow H_{1}(H, W) \rightarrow Y_{H} \rightarrow Z_{H} \rightarrow W_{H} \rightarrow 0 .
$$

If $H=\operatorname{Gal}\left(K_{\infty} / F\right)$ is cyclic, then the sequence

$$
0 \rightarrow Y^{H} \rightarrow Z^{H} \rightarrow W^{H} \rightarrow Y_{H} \rightarrow Z_{H} \rightarrow W_{H} \rightarrow 0
$$

is exact.

Proof. The first sequence is just part of the long exact homology sequence. The second is a straightforward consequence of the Snake Lemma.

LEMma 1.5. If $M$ is a finitely generated torsion-free $\Lambda$-module of rank $\varrho$, then for any $f \in \Lambda, f \neq 0$, there is an exact sequence

$$
0 \rightarrow M \rightarrow \Lambda^{\varrho} \rightarrow N \rightarrow 0,
$$

such that $N$ is a torsion $\Lambda$-module with an annihilator $g$ such that $(g, f)=1$, where $(g, f)$ is the greatest common divisor of $g$ and $f$.

Proof. Let $\Lambda_{f}=\{a / b: a$ and $b \in \Lambda,(b, f)=1\}$. Since $\Lambda_{f}$ is a principal ideal domain, $M \otimes \Lambda_{f}$ is a free $\Lambda_{f}$-module. The lemma follows.

Lemma 1.6. Let $s=\sum_{\wp \in S}\left[K_{\wp}: \mathbb{Q}_{p}\right]-r_{1}-r_{2}$. If $L / K$ is a finite extension such that $L \subset K_{\infty}$, let $S_{1}=\{q: q$ is a prime in $L$, and there 
exists $\wp \in S$ such that $q \mid \wp\}$ and let $s(L)=\sum_{q \in S_{1}}\left[F_{q}: \mathbb{Q}_{p}\right]-r_{1}(L)-r_{2}(L)$. Then $s(L)=s[L: K]$.

Proof. Because $L / K$ is unramified outside of $p$, we have $r_{1}(L)=$ $[L: K] r_{1}$ and $r_{2}(L)=[L: K] r_{2}$. Also for each $\wp \in S, \sum_{q \mid \wp, q \subset L}\left[F_{q}: \mathbb{Q}_{p}\right]=$ $[L: K]$. It follows that $s(L)=s[L: K]$.

From now on, we assume that for every $\mathbb{Z}_{p}$-extension $F$ of $K$ such that $F \subset K_{\infty}$, the weak Leopoldt hypothesis holds for $F$ and $S$. Fix such an $F$. Then for any field $L$ between $K$ and $F$, by class field theory and Lemma 1.6, $\operatorname{rank}_{\mathbb{Z}_{p}} X(L)=[L: K] s+\delta_{L}$. Since $\delta_{L}$ is bounded, if $s$ were negative, then we could choose an $L$ such that $[L: K]$ is large enough that $\operatorname{rank}_{\mathbb{Z}_{p}} X(L)=$ $[L: K] s+\delta_{L}$ is negative, which is a contradiction. Therefore, $s \geq 0$.

TheOrem 1.7. Let $S$ be as above. Then

(i) $I(\mathcal{G}) \operatorname{coker}\left(\pi_{X}\right)=0$ and $I(\mathcal{G}) I_{T(F)-S} \operatorname{ker}\left(\pi_{X}\right)=0$. Furthermore, $\operatorname{coker}\left(\pi_{X}\right)=\operatorname{Gal}\left(F_{\infty} / F\right)$ where $F_{\infty}$ is the maximal extension of $F$ in $K_{\infty}$ which is unramified outside of $S$, and $\operatorname{ker}\left(\pi_{X}\right)$ is finitely generated over $\mathbb{Z}_{p}$ when $F / K$ is finite.

(ii) $I(\mathcal{G}) \operatorname{coker}\left(\pi_{A}\right)=0$ and $I(\mathcal{G}) I_{T(F)} \operatorname{ker}\left(\pi_{A}\right)=0$. Further, $\operatorname{coker}\left(\pi_{A}\right)$ $=\operatorname{Gal}\left(F_{\mathrm{unr}} / F\right)$ where $F_{\mathrm{unr}}$ is the maximal extension of $F$ in $K_{\infty}$ which is everywhere unramified, and $\operatorname{ker}\left(\pi_{A}\right)$ is finitely generated over $\mathbb{Z}_{p}$ when $F / K$ is finite.

Proof. For $K$ imaginary quadratic, this was proved by Rubin [9]. We follow his procedures.

Since $\operatorname{coker}\left(\pi_{X}\right)=\operatorname{Gal}\left(M_{S}(F) \cap K_{\infty} / F\right)$, assertion (i) for $\operatorname{coker}\left(\pi_{X}\right)$ is clear. Let $M_{1}$ be $M_{S}\left(K_{\infty}\right)^{I(F) X\left(K_{\infty}\right)}$ and let $M_{2}$ be the maximal abelian extension of $F$ in $M_{S}\left(K_{\infty}\right)$. Then $\operatorname{Gal}\left(M_{1} / K_{\infty}\right)=X\left(K_{\infty}\right) / I(F) X\left(K_{\infty}\right)$ and $\operatorname{ker}\left(\pi_{X}\right)=\operatorname{Gal}\left(M_{1} / K_{\infty} M_{S}(F)\right)$. From Lemma 1.3, it follows that $I(\mathcal{G})$ annihilates $\operatorname{Gal}\left(M_{1} / M_{2}\right)$. Next we consider $\operatorname{Gal}\left(M_{2} / K_{\infty} M_{S}(F)\right)$.

Since $\operatorname{Gal}\left(M_{2} / F\right)$ is abelian, we have

$$
\operatorname{Gal}\left(M_{2} / M_{S}(F)\right)=\prod_{v \in S^{\prime}} I_{v},
$$

where $S^{\prime}$ is the set of primes of $F$ lying above $T(F)-S$, and for each $v \in S^{\prime}, I_{v}$ is the inertia group of $v$ in $\operatorname{Gal}\left(M_{2} / F\right)$. If $T(F)-S$ is empty, then $M_{2}=M_{S}(F)$. For $v \in S^{\prime}$, we have $v \mid \wp$, where $\wp \in T(F)-S$. If $\gamma \in D_{\wp}$ then $\gamma v=v$, so that $\gamma^{-1} I_{v} \gamma=I_{v}$. Since $M_{2} / K_{\infty}$ is unramified above $v, I_{v}$ injects into $\operatorname{Gal}\left(K_{\infty} / F\right)$ and it follows that $\gamma-1$ annihilates $I_{v}$. Thus $I\left(D_{\wp}\right)$ annihilates $I_{v}$. This means $I_{T(F)-S}$ annihilates $\operatorname{Gal}\left(M_{2} / M_{S}(F)\right)$.

Finally, we prove that $\operatorname{ker}\left(\pi_{X}\right)$ is finitely generated over $\mathbb{Z}_{p}$ when $F / K$ is finite. By Lemma $1.3, \operatorname{Gal}\left(M_{1} / M_{2}\right)$ is finitely generated over $\mathbb{Z}_{p}$. Now 
by the properties of $\left\{I_{v}\right\}_{v \in S^{\prime}}$ proved above and since $\operatorname{Gal}\left(M_{2} / M_{S}(F)\right)=$ $\prod_{v \in S^{\prime}} I_{v}$, we find that $\operatorname{Gal}\left(M_{2} / M_{S}(F)\right)$ is finitely generated over $\mathbb{Z}_{p}$. Because $\operatorname{ker}\left(\pi_{X}\right)=\operatorname{Gal}\left(M_{1} / K_{\infty} M_{S}(F)\right)$, we have proved (i).

The proof of (ii) is exactly the same as the proof of (i), except that $X\left(K_{\infty}\right), M_{S}\left(K_{\infty}\right)$ and $M_{S}(F)$ need to be changed into $A\left(K_{\infty}\right), L\left(K_{\infty}\right)$ and $L(F)$, where $L\left(K_{\infty}\right)$ (resp. $L(F)$ ) is the maximal abelian unramified p-extension of $K_{\infty}$ (resp. $F$ ).

TheOREM 1.8. Assume that for every $\mathbb{Z}_{p}$-extension $F$ of $K$ such that $F \subset K_{\infty}$, the weak Leopoldt hypothesis holds for $F$ and $S$. Then $X\left(K_{\infty}\right)$ is a finitely generated $\Lambda$-module of rank s.

Pro of. For $K$ imaginary quadratic, this was proved by Rubin in Theorem 5.3(iii) of [9], and for $S=\{$ all $\wp$ above $p\}$ by Greenberg [3]. We basically follow [3].

If $F$ is a finite extension of $K$, then the exact sequence

$$
0 \rightarrow \operatorname{ker}\left(\pi_{X}\right) \rightarrow X\left(K_{\infty}\right)_{F} \rightarrow X(F)
$$

shows that, because $\operatorname{ker}\left(\pi_{X}\right)$ and $X(F)$ are finitely generated over $\mathbb{Z}_{p}$, so is $X\left(K_{\infty}\right)_{F}$. This implies that $X\left(K_{\infty}\right)$ is a finitely generated $\Lambda$-module. The statement about $\operatorname{rank}_{\Lambda} X\left(K_{\infty}\right)$ can be proved by induction. We shall use $\tau$ to denote $\operatorname{rank}_{\Lambda} X\left(K_{\infty}\right)$. Let $Y$ be the torsion $\Lambda$-submodule of $X\left(K_{\infty}\right)$ and let $Z=X\left(K_{\infty}\right) / Y$. We use induction on $d$ to prove $\tau=s$.

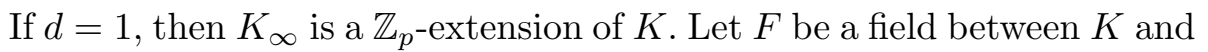
$K_{\infty}$. Let $M(F)$ be the maximal abelian extension of $F$ contained in $M_{S}\left(K_{\infty}\right)$ so it corresponds to the commutator subgroup of $\operatorname{Gal}\left(M_{S}\left(K_{\infty}\right) / F\right)$. Thus

$$
\operatorname{rank}_{\mathbb{Z}_{p}}\left(X\left(K_{\infty}\right) / I(F) X\left(K_{\infty}\right)\right)=\operatorname{rank}_{\mathbb{Z}_{p}} \operatorname{Gal}\left(M(F) / K_{\infty}\right) .
$$

By the same argument as in the proof of Theorem 1.7(i), we find that $\xi_{F}=$ $\operatorname{rank}_{\mathbb{Z}_{p}} \operatorname{Gal}\left(M(F) / M_{S}(F)\right)$ is bounded by a number independent of $F$, and

$$
\begin{aligned}
\operatorname{rank}_{\mathbb{Z}_{p}}\left(X\left(K_{\infty}\right) / I(F) X\left(K_{\infty}\right)\right) & =\operatorname{rank}_{\mathbb{Z}_{p}} \operatorname{Gal}\left(M_{S}(F) / K_{\infty}\right)+\xi_{F} \\
& =\operatorname{rank}_{\mathbb{Z}_{p}} X(F)-1+\xi_{F} .
\end{aligned}
$$

However, $\operatorname{rank}_{\mathbb{Z}_{p}} X(F)=[F: K] s+\delta_{F}$. Thus

$$
\operatorname{rank}_{\mathbb{Z}_{p}}\left(X\left(K_{\infty}\right) / I(F) X\left(K_{\infty}\right)\right)=[F: K] s-1+\xi_{F}+\delta_{F} .
$$

On the other hand, it follows from the structure theory of $\Lambda$-modules that

$$
\operatorname{rank}_{\mathbb{Z}_{p}}\left(X\left(K_{\infty}\right) / I(F) X\left(K_{\infty}\right)\right)=\tau[F: K]+\varepsilon_{F},
$$

where $\varepsilon_{F}=\operatorname{rank}_{\mathbb{Z}_{p}}(Y / I(F) Y)$, so it is bounded. We now have $[F: K] s-1+$ $\xi_{F}+\delta_{F}=\tau[F: K]+\varepsilon_{F}$, which means $\tau=s$, since $\delta_{F}$ is bounded because of the weak Leopoldt hypothesis. This proves that $\tau=s$ when $d=1$. 
If $d \geq 2$, we assume that the conclusion is true for $d-1$. Let $H$ be a direct summand of $\mathcal{G}$ isomorphic to $\mathbb{Z}_{p}$ and let $h$ be a topological generator of $H$. From the exact sequence $0 \rightarrow Y \rightarrow X\left(K_{\infty}\right) \rightarrow Z \rightarrow 0$ and Lemma 1.4 , we get

$$
0 \rightarrow Y_{H} \rightarrow X\left(K_{\infty}\right)_{H} \rightarrow Z_{H} \rightarrow 0,
$$

since $Z^{H}=0$. This implies

$$
\operatorname{rank}_{\Lambda_{H}} X\left(K_{\infty}\right)_{H}=\operatorname{rank}_{\Lambda_{H}}\left(Z_{H}\right)+\operatorname{rank}_{\Lambda_{H}}\left(Y_{H}\right) .
$$

But from Lemma 1.5, we have an exact sequence

$$
0 \rightarrow Z \rightarrow \Lambda^{\tau} \rightarrow N \rightarrow 0,
$$

in which $N$ has an annihilator $g$ such that $(g, h-1)=1$. This gives us the exact sequence

$$
N^{H} \rightarrow Z_{H} \rightarrow \Lambda_{H}^{\tau} \rightarrow N_{H} \rightarrow 0 .
$$

Since the image of $g$ in $\Lambda_{H}$, which is not zero, annihilates $N_{H}$ and $N^{H}$, we know that $\operatorname{rank}_{\Lambda_{H}}\left(Z_{H}\right)=\tau$. Combining the above, we get

$$
\operatorname{rank}_{\Lambda_{H}} X\left(K_{\infty}\right)_{H}=\tau+\operatorname{rank}_{\Lambda_{H}}\left(Y_{H}\right) .
$$

Let $\Phi \in \Lambda$ be a nonzero annihilator of $Y$ and for all $\wp \in T\left(K^{\prime}\right)-S$ such that $D_{\wp}$ is cyclic, let $h_{\wp}$ be a topological generator of $D_{\wp}$. The fixed field of $H$ will be denoted by $K^{\prime}$. We choose $H$ so that $h-1$ does not divide $\Phi$ or $h_{\wp}-1$ for all $\wp \in T\left(K^{\prime}\right)-S$ such that $D_{\wp}$ is cyclic. For such $H, Y_{H}$ is a torsion $\Lambda_{H}$-module, since the projection $\bar{\Phi}$ of $\Phi$ in $\Lambda_{H}$ is a nonzero annihilator of $Y_{H}$. Hence $\operatorname{rank}_{\Lambda_{H}} X\left(K_{\infty}\right)_{H}=\tau$. Now we consider the following exact sequence of $\Lambda_{H}$-modules:

$$
0 \rightarrow \operatorname{ker}\left(\pi_{X}\right) \rightarrow X\left(K_{\infty}\right)_{H} \rightarrow X\left(K^{\prime}\right) \rightarrow \operatorname{coker}\left(\pi_{X}\right) \rightarrow 0 .
$$

Because of the way $H$ was chosen, there exists $\alpha \in I_{T\left(K^{\prime}\right)-S}$ such that $\alpha$ is not a zero divisor in $\Lambda_{H}$. Since $I(\mathcal{G}) I_{T\left(K^{\prime}\right)-S} \operatorname{ker}\left(\pi_{X}\right)=0$ and $I(\mathcal{G}) \operatorname{coker}\left(\pi_{X}\right)$ $=0$, we conclude that both $\operatorname{ker}\left(\pi_{X}\right)$ and $\operatorname{coker}\left(\pi_{X}\right)$ are torsion $\Lambda_{H}$-modules. This means

$$
\tau=\operatorname{rank}_{\Lambda_{H}} X\left(K_{\infty}\right)_{H}=\operatorname{rank}_{\Lambda_{H}} X\left(K^{\prime}\right) .
$$

By the induction hypothesis, $\operatorname{rank}_{\Lambda_{H}} X\left(K^{\prime}\right)=s$. This completes the proof of Theorem 1.8.

2. Results about $X(F)$ and $A(F)$. Let $\mu_{p^{\infty}}$ be the discrete group of all $p$-power roots of unity. We denote by $\mathcal{X}$ the set of continuous characters $\varrho: \mathcal{G} \rightarrow \mu_{p}$. Every $\varrho \in \mathcal{X}$ extends uniquely to a continuous homomorphism on $\Lambda$. For $f \in \Lambda$, define $\mathcal{X}(f)=\{\varrho \in \mathcal{X}: \varrho(f)=0\}$. Let $\gamma_{1}, \ldots, \gamma_{d}$ be fixed topological generators of $\mathcal{G}$. We define an injection from $\mathcal{X}(f)$ to $\mu_{p^{\infty}}^{d}$ by mapping $\varrho \in \mathcal{X}(f)$ to $\left(\varrho\left(\gamma_{1}\right), \ldots, \varrho\left(\gamma_{d}\right)\right)$. This identifies $\mathcal{X}(f)$ with the set 
of zeros of $f$ in $\left(\mu_{p^{\infty}}\right)^{d}$. Also, $I(f)$ will represent the set $\{g \in \Lambda: \varrho(g)=$ 0 for all $\varrho \in \mathcal{X}(f)\}$. Following Monsky [8], we let $E_{d}$ be the free rank $d$

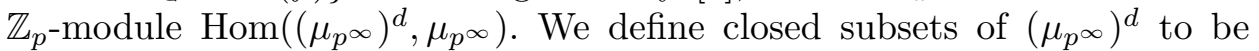
the subsets that are finite unions of subsets of $\left(\mu_{p^{\infty}}\right)^{d}$ each of which is defined by a set of equations $\tau_{j}(\zeta)=\epsilon_{j}$, where $\tau_{j} \in E_{d}, \zeta \in\left(\mu_{p^{\infty}}\right)^{d}, \epsilon_{j} \in$

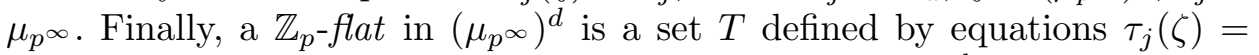
$\epsilon_{j}$, where $\left\{\tau_{j}\right\}$ is a subset of a basis of $E_{d}, \zeta \in\left(\mu_{p^{\infty}}\right)^{d}$, and $\epsilon_{j} \in \mu_{p^{\infty}}$. Suppose $\left\{\tau_{j}: 1 \leq j \leq d\right\}$ is a basis of $E_{d}$ and $T$ is defined by $\tau_{j}$ for all $j$ such that $1 \leq j \leq k$. Then we say that the dimension of $T$ is $d-k$. Theorem 2.6 of [8] implies that $\mathcal{X}(f)$, as a subset of $\left(\mu_{p^{\infty}}\right)^{d}$, is closed. This means $\mathcal{X}(f)$ is a finite union of $\mathbb{Z}_{p}$-flats. We write $\operatorname{dim} \mathcal{X}(f) \leq \alpha$ if there is a finite set $\left\{U_{i}\right\}$ of $\mathbb{Z}_{p}$-flats such that $\bigcup_{i} U_{i}$ covers $\mathcal{X}(f)$ and $\operatorname{dim} U_{i} \leq \alpha$ for all $i$.

Lemma 2.1. Suppose $d \geq 2$ and $f \in \Lambda$.

(i) If $\operatorname{dim} \mathcal{X}(f) \leq d-2$, then $I(f)$ is an ideal of height at least two.

(ii) If $f$ is relatively prime to $\gamma-1$ for every $\gamma \neq 1$ in $\mathcal{G}$, then $\operatorname{dim} \mathcal{X}(f) \leq$ $d-2$.

(iii) Let $g$ be a prime in $\Lambda$ such that $\mathcal{X}(g)$ has codimension 1 . There exists a field $F$ such that $K \subset F \subset K_{\infty}$ and $H=\operatorname{Gal}\left(K_{\infty} / F\right) \cong \mathbb{Z}_{p}$, with the property $g \mid h-1$, where $h$ is a topological generator of $H$.

Proof. (i) Since $\operatorname{dim} \mathcal{X}(f) \leq d-2, \mathcal{X}(f)$ can be written as $\bigcup_{i=1}^{m} T_{i}$, where $m$ is a positive integer and for all $i, 1 \leq i \leq m, T_{i}$ is a $\mathbb{Z}_{p}$-flat such that $\operatorname{dim} T_{i} \leq d-2$. It follows that for each $i, 1 \leq i \leq m$, there exist $f_{i}, g_{i} \in \Lambda$ such that $\left(f_{i}, g_{i}\right)=1$ and $T_{i} \subset \mathcal{X}\left(f_{i}\right) \cap \mathcal{X}\left(g_{i}\right)$. Let $\mathcal{A}_{i}$ be the ideal generated by $f_{i}$ and $g_{i}, 1 \leq i \leq m$. Then $\prod_{i=1}^{m} \mathcal{A}_{i} \subset I(f)$ and $\prod_{i=1}^{m} \mathcal{A}_{i}$ is an ideal of height at least two in $\Lambda$. This means $I(f)$ is an ideal of height at least two in $\Lambda$.

(ii) can be deduced from Theorem 2.6 of [7].

(iii) By (ii), we could get a $\gamma \in \mathcal{G}$ such that $(g, \gamma-1) \neq 1$. Since $g$ is prime, $g \mid \gamma-1$. Let $F$ be the fixed field of $\gamma$. Then $H=\operatorname{Gal}\left(K_{\infty} / F\right)$ is generated by $\gamma$ topologically. This completes the proof of (iii).

TheOREM 2.2. Let $g$ be a prime element in $\Lambda$. Let $F$ be any field such that $K \subset F \subset K_{\infty}$ and $H=\operatorname{Gal}\left(K_{\infty} / F\right) \cong \mathbb{Z}_{p}$. If $g \mid h-1$, where $h$ is a topological generator of $H$, then $\operatorname{rank}_{\Lambda / g \Lambda}(X(F) \otimes(\Lambda / g \Lambda))=s$.

Proof. Let $G^{\prime}$ be a direct summand of $\mathcal{G}$ such that $G^{\prime} \cong \mathbb{Z}_{p}$ and $H \subset G^{\prime}$. We can now write $\operatorname{Gal}(F / K)$ as $V \oplus G^{\prime \prime}$, where $G^{\prime \prime} \cong \mathbb{Z}_{p}^{d-1}$ and $V \cong G^{\prime} / H$. Denote by $L$ the fixed field of $G^{\prime \prime}$ and by $K^{\prime}$ the fixed field of $V$. Let $g^{\prime}$ be a topological generator of $G^{\prime}$, and let $\Lambda^{\prime}$ be the Iwasawa algebra $\mathbb{Z}_{p}\left[\left[G^{\prime \prime}\right]\right]$. 


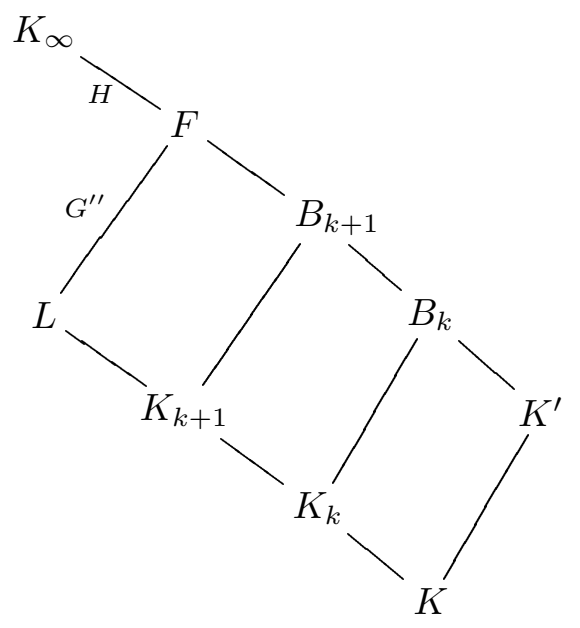

Since $g$ is a prime and $g \mid h-1, g=\omega_{k+1} / \omega_{k}$, where $k$ is a positive integer and $\omega_{j}=g^{\prime p^{j}}-1$ for $j=k, k+1$. For the field $K_{i}$ between $K$ and $L$ corresponding to $g^{\prime p^{i}}$, let $B_{i}$ be $K^{\prime} K_{i}$. Since $\operatorname{Gal}\left(K^{\prime} K_{i} / K_{i}\right) \cong G^{\prime \prime}, X\left(B_{i}\right)$ can be considered as a $\Lambda^{\prime}$-module. Consider the exact sequence

$$
0 \rightarrow \operatorname{ker}\left(\pi_{X\left(B_{i}\right)}\right) \rightarrow X(F) / I\left(B_{i}\right) X(F) \rightarrow X\left(B_{i}\right) \rightarrow \operatorname{coker}\left(\pi_{X\left(B_{i}\right)}\right) \rightarrow 0,
$$

where the middle map is the natural projection $\pi_{X\left(B_{i}\right)}$. Let $T^{\prime}\left(B_{i}\right)$ be the primes of $K$ which ramify in $F / B_{i}$. Write $M_{2}\left(B_{i}\right)$ for the maximal abelian extension of $B_{i}$ in $M_{S}(F)$. $S^{\prime \prime}$ will denote the set of primes of $B_{i}$ lying above $T^{\prime}\left(B_{i}\right)-S$. From the proof of Theorem 1.7(i), we find that coker $\left(\pi_{X\left(B_{i}\right)}\right)$ is finite, and that $\operatorname{ker}\left(\pi_{X\left(B_{i}\right)}\right)$ is a torsion $\Lambda^{\prime}$-module if $\prod_{v \in S^{\prime \prime}} I_{v}$ is a torsion $\Lambda^{\prime}$-module, where $I_{v}$ is the inertia group of $v$ in $\operatorname{Gal}\left(M_{2}\left(B_{i}\right) / B_{i}\right)$, and $I_{v}$ can be embedded into $\operatorname{Gal}\left(F / B_{i}\right)$. Since $\operatorname{Gal}\left(F / B_{i}\right)$ is finite, there exists a positive integer $j$ such that $p^{j} I_{v}=0$ for all $v \in S^{\prime \prime}$, which means $p^{j} \prod_{v \in S^{\prime \prime}} I_{v}=0$.

This means $\operatorname{rank}_{\Lambda^{\prime}}\left(X(F) / I\left(B_{i}\right) X(F)\right)=\operatorname{rank}_{\Lambda^{\prime}} X\left(B_{i}\right)$. By Lemma 1.6 and Theorem 1.8, $\operatorname{rank}_{\Lambda^{\prime}}\left(X(F) / I\left(B_{i}\right) X(F)\right)=\operatorname{rank}_{\Lambda^{\prime}} X\left(B_{i}\right)=s p^{i}$.

Next consider the exact sequence

$$
\begin{aligned}
0 \rightarrow I\left(B_{k}\right) X(F) / I\left(B_{k+1}\right) X(F) \rightarrow X(F) / I( & \left.B_{k+1}\right) X(F) \\
& \rightarrow X(F) / I\left(B_{k}\right) X(F) \rightarrow 0 .
\end{aligned}
$$

Since

$$
I\left(B_{k}\right) X(F) / I\left(B_{k+1}\right) X(F)=\omega_{k} X(F) / \omega_{k+1} X(F)=\omega_{k} X(F) / g \omega_{k} X(F),
$$

we have

$$
\operatorname{rank}_{\Lambda^{\prime}}\left(\omega_{k} X(F) / g \omega_{k} X(F)\right)=s\left(p^{k+1}-p^{k}\right) .
$$

Claim. $\omega_{k} X(F) / g \omega_{k} X(F)$ and $X(F) / g X(F)$ have the same rank as $\Lambda / g \Lambda$-modules. 
If the claim is true, then since $\Lambda^{\prime}$ can be embedded into $\Lambda / g \Lambda$ and $\operatorname{rank}_{\Lambda^{\prime}}(\Lambda / g \Lambda)=p^{k+1}-p^{k}$, we have

$$
\operatorname{rank}_{\Lambda / g \Lambda}(X(F) \otimes(\Lambda / g \Lambda))=\operatorname{rank}_{\Lambda / g \Lambda}(X(F) / g X(F))=s .
$$

This would complete the proof of the theorem.

To prove the claim, we consider the commutative diagram

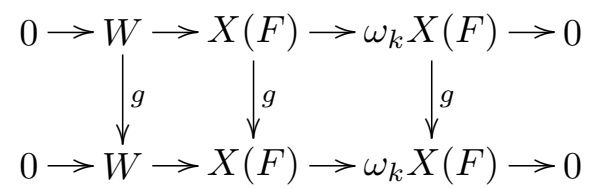

where $W$ is the kernel of multiplication by $\omega_{k}$ and the vertical maps are multiplications by $g$. By the Snake Lemma, we get the exact sequence

$$
W / g W \rightarrow X(F) / g X(F) \rightarrow \omega_{k} X(F) / g \omega_{k} X(F) \rightarrow 0 .
$$

Since $\omega_{k}$ is not a zero divisor in $\Lambda / g \Lambda$ and $\omega_{k}(W / g W)=0$, we have proved the claim.

From now on, assume that for any field $F$ between $K$ and $K_{\infty}$ such that $F$ is finite over $K$, Leopoldt's conjecture holds for $F$.

According to the classification theorem, for any torsion $\Lambda$-module $Y$, we have exact sequences

$$
\begin{gathered}
0 \rightarrow \bigoplus \Lambda / f_{i} \Lambda \rightarrow Y \rightarrow N \rightarrow 0, \\
0 \rightarrow N_{1} \rightarrow Y \rightarrow \bigoplus \Lambda / f_{i} \Lambda \rightarrow N_{2} \rightarrow 0,
\end{gathered}
$$

in which $f_{i} \in \Lambda$ for all $i$ and $N, N_{1}, N_{2}$ can be annihilated by an ideal of height at least two in $\Lambda$. We call the ideal generated by $\prod f_{i}$ the characteristic ideal of $Y$, written $\operatorname{char}(Y)$.

\section{Preliminary results}

Proposition 3.1. (i) If $f \in \Lambda$ and $H \subset \mathcal{G}$, then $I(f) H_{1}(H, \Lambda / f \Lambda)$ $=0$.

(ii) If $Y$ is a finitely generated torsion $\Lambda$-module, then there is an ideal $\mathcal{B}$ of height at least two in $\Lambda$ such that for any $H \subset \mathcal{G}, \mathcal{B} I(\operatorname{char}(Y)) H_{1}(H, Y)$ $=0$.

Proof. For $K$ imaginary quadratic, this was proved by Rubin in [9], Lemma 7.3. The same argument can be used here.

Proposition 3.2. Suppose $d \geq 2$. Let $Y=X\left(K_{\infty}\right)_{\text {torsion }}$ be the torsion submodule of the $\Lambda$-module $X\left(K_{\infty}\right)$. There is an ideal $\mathcal{C}$ of height at least two in $\Lambda$ such that $\mathcal{C} I_{T(K)} \subset I(\operatorname{char}(Y))$ and $\mathcal{C} I_{T(K)} \subset I\left(\operatorname{char}\left(A\left(K_{\infty}\right)\right)\right)$. 
Proof. It follows from Theorem 1 of [2] and Lemma 2.1 and Theorem 2.2 .

Proposition 3.3. There is an ideal $\mathcal{B} \subset \Lambda$ of height at least two, such that for every $H \subset \operatorname{Gal}\left(K_{\infty} / K\right)$,

$$
I_{T(K)} \mathcal{B} H_{1}\left(H, U\left(K_{\infty}\right) / \overline{\mathcal{E}}\left(K_{\infty}\right)\right)=0 \quad \text { and } \quad I_{T(K)} \mathcal{B} H_{1}\left(H, A\left(K_{\infty}\right)\right)=0 .
$$

Pr o of. When $K$ is imaginary quadratic and $d=2$, this is Corollary 7.5 of $[9]$.

By the inclusion $U\left(K_{\infty}\right) / \overline{\mathcal{E}}\left(K_{\infty}\right) \subset X\left(K_{\infty}\right)$ of global class field theory, $\left(U\left(K_{\infty}\right) / \overline{\mathcal{E}}\left(K_{\infty}\right)\right)_{\text {torsion }} \subset Y$. If $U\left(K_{\infty}\right) / \overline{\mathcal{E}}\left(K_{\infty}\right)$ is torsion, we can use Propositions 3.2 and 3.1 to get $I_{T(K)} \mathcal{B} H_{1}\left(H, U\left(K_{\infty}\right) / \overline{\mathcal{E}}\left(K_{\infty}\right)\right)=0$. In general, there is an exact sequence

$$
0 \rightarrow\left(U\left(K_{\infty}\right) / \overline{\mathcal{E}}\left(K_{\infty}\right)\right)_{\text {torsion }} \rightarrow U\left(K_{\infty}\right) / \overline{\mathcal{E}}\left(K_{\infty}\right) \rightarrow Z \rightarrow 0,
$$

where, by the exact sequence $0 \rightarrow U\left(K_{\infty}\right) / \overline{\mathcal{E}}\left(K_{\infty}\right) \rightarrow X\left(K_{\infty}\right) \rightarrow A\left(K_{\infty}\right)$ $\rightarrow 0$ of global class field theory, and by Theorems 1.7(ii) and 1.8, $Z$ is a torsion-free $\Lambda$-module of rank $s$. Now by using Lemma 1.5, one could see that $H_{1}(H, Z)$ is pseudo-null. Now $I_{T(K)} \mathcal{B} H_{1}\left(H, U\left(K_{\infty}\right) / \overline{\mathcal{E}}\left(K_{\infty}\right)\right)=0$ follows from Proposition 3.2 and $I(\operatorname{char}(Y)) H_{1}\left(H, U\left(K_{\infty}\right) / \overline{\mathcal{E}}\left(K_{\infty}\right)\right.$ torsion $)=0$.

By Propositions 3.1 and 3.2, there is an ideal $\mathcal{B} \subset \Lambda$ of height at least two, such that $H_{1}\left(H, A\left(K_{\infty}\right)\right)$ is annihilated by $I_{T(K)} \mathcal{B}$. This proves the second equation.

4. Main theorems. From now on, if $M$ is a $\Lambda$-module, we denote $M / I(F) M$ by $M_{F}$.

Theorem 4.1. Suppose $F$ is any extension of $K$ contained in $K_{\infty}$. There is an ideal $\mathcal{A} \subset \Lambda$ of height at least two, independent of $F$, such that

$$
I_{T(K)}^{3} \mathcal{A} \operatorname{coker}\left(\pi_{\mathcal{E}}\right)=0 \quad \text { and } \quad I_{T(K)}^{d+1} \mathcal{A} \operatorname{ker}\left(\pi_{\mathcal{E}}\right)=0 .
$$

Proof. When $d=2$ and $K$ is an imaginary quadratic field, this was proved by Rubin in [9], Theorem 7.6(i).

Consider the two commutative diagrams with exact rows

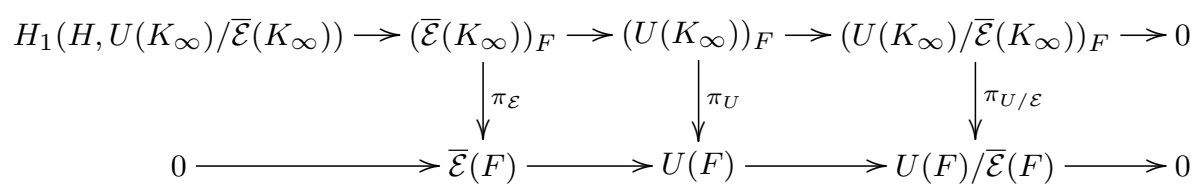


and

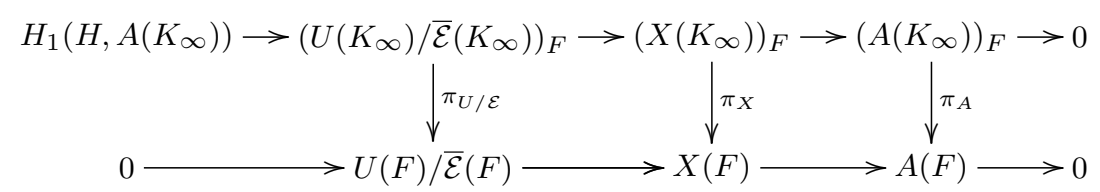

in which the top rows come from the exact sequences

$$
\begin{aligned}
0 & \rightarrow \overline{\mathcal{E}}\left(K_{\infty}\right) \rightarrow U\left(K_{\infty}\right) \rightarrow U\left(K_{\infty}\right) / \overline{\mathcal{E}}\left(K_{\infty}\right) \rightarrow 0, \\
0 & \rightarrow U\left(K_{\infty}\right) / \overline{\mathcal{E}}\left(K_{\infty}\right) \rightarrow X\left(K_{\infty}\right) \rightarrow A\left(K_{\infty}\right) \rightarrow 0 .
\end{aligned}
$$

By the Snake Lemma, we get the following exact sequences:

$$
\begin{aligned}
H_{1}\left(H, U\left(K_{\infty}\right) / \overline{\mathcal{E}}\left(K_{\infty}\right)\right) \rightarrow \operatorname{ker}\left(\pi_{\mathcal{E}}\right) & \rightarrow \operatorname{ker}\left(\pi_{U}\right) \rightarrow \operatorname{ker}\left(\pi_{U / \mathcal{E}}\right) \rightarrow \operatorname{coker}\left(\pi_{\mathcal{E}}\right) \\
& \rightarrow \operatorname{coker}\left(\pi_{U}\right) \rightarrow \operatorname{coker}\left(\pi_{U / \mathcal{E}}\right) \rightarrow 0
\end{aligned}
$$

and

$$
\begin{aligned}
H_{1}\left(H, A\left(K_{\infty}\right)\right) \rightarrow \operatorname{ker}\left(\pi_{U / \mathcal{E}}\right) & \rightarrow \operatorname{ker}\left(\pi_{X}\right) \rightarrow \operatorname{ker}\left(\pi_{A}\right) \rightarrow \operatorname{coker}\left(\pi_{U / \mathcal{E}}\right) \\
& \rightarrow \operatorname{coker}\left(\pi_{X}\right) \rightarrow \operatorname{coker}\left(\pi_{A}\right) \rightarrow 0 .
\end{aligned}
$$

Now the annihilator of $\operatorname{ker}\left(\pi_{\mathcal{E}}\right)$ comes from the annihilators of $\operatorname{ker}\left(\pi_{U}\right)$ (Theorem 1.2) and $H_{1}\left(H, U\left(K_{\infty}\right) / \overline{\mathcal{E}}\left(K_{\infty}\right)\right.$ ) (Proposition 3.3). Similarly we get the annihilator of $\operatorname{ker}\left(\pi_{U / \mathcal{E}}\right)$ from the annihilators of $\operatorname{ker}\left(\pi_{X}\right)$ (Theorem 1.7) and $H_{1}\left(H, A\left(K_{\infty}\right)\right.$ ) (Proposition 3.3), and then the annihilator of $\operatorname{coker}\left(\pi_{\mathcal{E}}\right)$ comes from that of $\operatorname{ker}\left(\pi_{U / \mathcal{E}}\right)$ and $\operatorname{coker}\left(\pi_{U}\right)$ (Theorem 1.2). This completes the proof of this theorem.

TheOREM 4.2. Assume that the Iwasawa- $\mu$-conjecture is true for $K$. Also assume that for any field $F$ between $K$ and $K_{\infty}$ such that $F$ is finite over $K$, Leopoldt's conjecture holds for $F$. Let $F, \mathcal{A}$ be as in Theorem 4.1 above. Then

$$
I_{T(K)}^{3} \mathcal{A}\left(\left(\mathcal{E}(F) / \mathcal{E}(F)^{\text {univ }}\right) \otimes \mathbb{Z}_{p}\right)=0 .
$$

Pr o of. When $d=1$, this result is due to Iwasawa ([5]).

If $F / K$ is a finite extension, it follows from Theorem 4.1 that

$$
\overline{\mathcal{E}}(F) / \bigcap_{L \subset K_{\infty}, L / F \text { finite }} N_{L / F}(\overline{\mathcal{E}}(L)) \cong \operatorname{coker}\left(\pi_{\mathcal{E}}\right)
$$

is annihilated by $I_{T(K)}^{3} \mathcal{A}$. Now from our assumption of Leopoldt's conjecture, we get

$$
I_{T(K)}^{3} \mathcal{A}\left(\mathcal{E}(F) \otimes \mathbb{Z}_{p} / \bigcap_{L \subset K_{\infty}, L / F \text { finite }} N_{L / F}\left(\mathcal{E}(L) \otimes \mathbb{Z}_{p}\right)\right)=0 .
$$

This implies

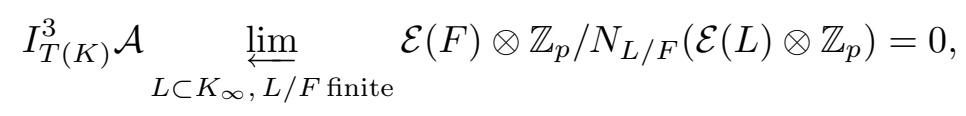


which implies

$$
I_{T(K)}^{3} \mathcal{A}{\underset{L \subset K \infty}{\longleftarrow}, L / F \text { finite }}_{\lim _{\infty}}\left(\mathcal{E}(F) / N_{L / F} \mathcal{E}(L)\right) \otimes \mathbb{Z}_{p}=0
$$

Now it is clear that $I_{T(K)}^{3} \mathcal{A}\left(\left(\mathcal{E}(F) / \mathcal{E}(F)^{\text {univ }}\right) \otimes \mathbb{Z}_{p}\right)=0$. We proved the conclusion in this case.

If $F / K$ is an infinite extension, then

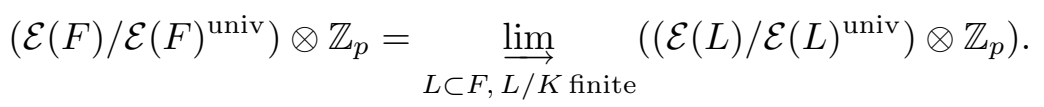

This proves the theorem.

Next we consider the cohomology group $H^{1}\left(\operatorname{Gal}\left(K_{\infty} / F\right), \mathcal{E}\left(K_{\infty}\right)\right)$. We first prove some results about $H^{1}(\operatorname{Gal}(B / F), \overline{\mathcal{E}}(B))$, where $B$ is a finite, cyclic extension of $F$ in $K_{\infty}$. Since $\pi_{\mathcal{E}}$ is dependent on $F$, we can write $\pi_{\mathcal{E}(F)}$ for $\pi_{\mathcal{E}}$ to indicate this dependence.

Proposition 4.3. Suppose $B$ is a finite, cyclic extension of $F$ in $K_{\infty}$. Let $\pi^{\prime}$ be the natural map

$$
\pi^{\prime}: \overline{\mathcal{E}}(B) / I\left(\operatorname{Gal}\left(K_{\infty} / F\right)\right) \overline{\mathcal{E}}(B) \rightarrow \overline{\mathcal{E}}(F),
$$

which is induced by the norm map. Then there exists an ideal $\mathcal{A}$ of height at least two in $\Lambda$, independent of $B$ and $F$, such that $I_{T(K)}^{d+4} \mathcal{A} \operatorname{ker}\left(\pi^{\prime}\right)=0$.

Proof. If we let $\phi$ be the natural projection

$$
\phi: \overline{\mathcal{E}}(B) \rightarrow \overline{\mathcal{E}}(B) / I\left(\operatorname{Gal}\left(K_{\infty} / F\right)\right) \overline{\mathcal{E}}(B),
$$

then $\pi_{\mathcal{E}(F)}=\pi^{\prime} \circ \phi \circ \pi_{\mathcal{E}(B)}$ and for any $\xi \in \operatorname{ker}\left(\pi^{\prime}\right)$, there exists $\eta \in \mathcal{E}(B)$ such that $\phi(\eta)=\xi$. Now from Theorem 4.1, there exists an ideal $\mathcal{B}$ of height at least two in $\Lambda$ such that $I_{T(K)}^{3} \mathcal{B} \operatorname{coker}\left(\pi_{\mathcal{E}(B)}\right)=0$. This means for any $\alpha \in I_{T(K)}^{3} \mathcal{B}$, there exists $\zeta \in \overline{\mathcal{E}}\left(K_{\infty}\right) / I(B) \overline{\mathcal{E}}\left(K_{\infty}\right)$ such that $\alpha \eta=$ $\pi_{\mathcal{E}(B)}(\zeta)$. From this, we get $\alpha \xi=\phi(\alpha \eta)=\phi\left(\pi_{\mathcal{E}(B)}(\zeta)\right)$, which implies $\pi^{\prime} \circ$ $\phi \circ \pi_{\mathcal{E}(B)}(\zeta)=0$, from which we get $\pi_{\mathcal{E}(F)}(\zeta)=0$. From Theorem 4.1 again, $I_{T(K)}^{d+1} \mathcal{B} \operatorname{ker}\left(\pi_{\mathcal{E}(F)}\right)=0$. This means $\beta \zeta=0$ for any $\beta \in I_{T(K)}^{d+1} \mathcal{B}$, which implies $\alpha \beta \eta=\pi_{\mathcal{E}(B)}(\beta \zeta)=0$. This yields $\alpha \beta \xi=\phi(\alpha \beta \eta)=0$. The proof is complete.

Proposition 4.4. Let $B, F$ and $\pi^{\prime}$ be as in Proposition 4.3. Then

$$
\operatorname{ker}\left(\pi^{\prime}\right)=H^{1}(\operatorname{Gal}(B / F), \overline{\mathcal{E}}(B)) .
$$

Proof. By the definition of $\pi^{\prime}$ and by the definition before Theorem 3 in Chapter IV of [1], we get $\operatorname{ker}\left(\pi^{\prime}\right)=\widehat{H}^{-1}(\operatorname{Gal}(B / F), \overline{\mathcal{E}}(B))$ and

$$
\widehat{H}^{1}(\operatorname{Gal}(B / F), \overline{\mathcal{E}}(B))=H^{1}(\operatorname{Gal}(B / F), \overline{\mathcal{E}}(B)) .
$$

Since $\operatorname{Gal}(B / F)$ is cyclic, by Theorem 5 in Chapter IV of [1], we get

$$
\widehat{H}^{-1}(\operatorname{Gal}(B / F), \overline{\mathcal{E}}(B))=\widehat{H}^{1}(\operatorname{Gal}(B / F), \overline{\mathcal{E}}(B)) .
$$


Combining the above gives $\operatorname{ker}\left(\pi^{\prime}\right)=H^{1}(\operatorname{Gal}(B / F), \overline{\mathcal{E}}(B))$. This completes the proof.

Corollary 4.5. Suppose $F$ is a finite extension over $K$ and suppose $B \subset K_{\infty}$ is finite and cyclic over $F$. Then there exists an ideal $\mathcal{A}$ of height at least two in $\Lambda$, independent of $B$ and $F$, such that

$$
I_{T(K)}^{d+4} \mathcal{A} H^{1}(\operatorname{Gal}(B / F), \mathcal{E}(B))=0 .
$$

Pro of. Combining Propositions 4.3 and 4.4, we get

$$
I_{T(K)}^{d+4} \mathcal{A} H^{1}(\operatorname{Gal}(B / F), \overline{\mathcal{E}}(B))=0 .
$$

Since $F / K$ is a finite extension, the extension $B / K$ is also finite. This implies $H^{1}(\operatorname{Gal}(B / F), \overline{\mathcal{E}}(B))=H^{1}\left(\operatorname{Gal}(B / F), \mathcal{E}(B) \otimes \mathbb{Z}_{p}\right)$ by our assumption of Leopoldt's conjecture. Now since $\operatorname{Gal}(B / F)$ is a $p$-group, we get

$$
H^{1}(\operatorname{Gal}(B / F), \mathcal{E}(B)) \cong H^{1}\left(\operatorname{Gal}(B / F), \mathcal{E}(B) \otimes \mathbb{Z}_{p}\right),
$$

as $\Lambda$-modules. This shows that

$$
I_{T(K)}^{d+4} \mathcal{A} H^{1}(\operatorname{Gal}(B / F), \mathcal{E}(B))=0 .
$$

Theorem 4.6. Assume that the Iwasawa- $\mu$-conjecture is true for $K$. Also assume that for any field $F$ between $K$ and $K_{\infty}$ such that $F$ is finite over $K$, Leopoldt's conjecture holds for $F$. Suppose $F$ is a field such that $K \subset F$ $\subset K_{\infty}$. There exists an ideal $\mathcal{A}$ of height at least two in $\Lambda$, independent of $F$, such that

$$
I_{T(K)}^{d(d+4)} \mathcal{A} H^{1}\left(\operatorname{Gal}\left(K_{\infty} / F\right), \mathcal{E}\left(K_{\infty}\right)\right)=0 .
$$

Proof. When $d=1$, this result is due to Iwasawa ([5]).

First we assume that $F / K$ is a finite extension. Since

$$
H^{1}\left(\operatorname{Gal}\left(K_{\infty} / F\right), \mathcal{E}\left(K_{\infty}\right)\right)=\underset{B \subset K_{\infty}, B / F \text { finite }}{\underset{\lim _{\longrightarrow}}{\longrightarrow}} H^{1}(\operatorname{Gal}(B / F), \mathcal{E}(B)),
$$

we only need to show $I_{T(K)}^{d(d+4)} \mathcal{A} H^{1}(\operatorname{Gal}(B / F), \mathcal{E}(B))=0$ when $B \subset K_{\infty}$ and $B / F$ is a finite extension.

Since $K_{\infty} / K$ is a $\mathbb{Z}_{p}^{d}$-extension, $\operatorname{Gal}(B / F)$ is a product of $m$ cyclic factors, where $m$ is an integer, $m \leq d$. If $m=0, \operatorname{Gal}(B / F)$ is trivial, so we can assume $1 \leq m \leq d$.

We use induction on $m$ to prove $I_{T(F)}^{m(d+4)} \mathcal{A} H^{1}(\operatorname{Gal}(B / F), \mathcal{E}(B))=0$.

If $m=1$, then $B / F$ is a cyclic extension. From Corollary 4.5, there exists an ideal $\mathcal{A}$ of height at least two in $\Lambda$, independent of $F$ and $B$, such that $I_{T(K)}^{d+4} \mathcal{A} H^{1}(\operatorname{Gal}(B / F), \mathcal{E}(B))=0$.

Suppose the conclusion is true for $m-1$, that is, if $\operatorname{Gal}(B / F)$ is a product of $m-1$ cyclic factors, then there exists an ideal $\mathcal{B}$ of height at least two in $\Lambda$, independent of $F$ and $B$, such that $I_{T(K)}^{(m-1)(d+4)} \mathcal{B} H^{1}(\operatorname{Gal}(B / F), \mathcal{E}(B))=0$. 
Now if $\operatorname{Gal}(B / F)$ is a product of $m$ cyclic factors, we let $H$ be a subgroup of $\operatorname{Gal}(B / F)$ such that $H$ is a product of $m-1$ cyclic factors, and $\operatorname{Gal}(B / F) / H$ is cyclic. Let $C$ be the fixed field of $H$. Then the restriction-inflation sequence gives us the exact sequence

$$
\begin{aligned}
0 \rightarrow H^{1}(\operatorname{Gal}(C / F), \mathcal{E}(C)) \rightarrow H^{1}(\operatorname{Gal}(B / F), \mathcal{E}(B)) & \\
& \rightarrow H^{1}(\operatorname{Gal}(B / C), \mathcal{E}(B)) .
\end{aligned}
$$

Since $\operatorname{Gal}(C / F)$ is cyclic, we have an ideal $\mathcal{C}$ of height at least two in $\Lambda$, independent of $F$ and $C$, such that $I_{T(K)}^{d+4} \mathcal{C} H^{1}(\operatorname{Gal}(C / F), \mathcal{E}(C))=0$. As for $H^{1}(\operatorname{Gal}(B / C), \mathcal{E}(B))$, the induction hypothesis implies

$$
I_{T(K)}^{(m-1)(d+4)} \mathcal{B} H^{1}(\operatorname{Gal}(B / C), \mathcal{E}(B))=0 .
$$

Combining these we get

$$
I_{T(K)}^{m(d+4)} \mathcal{B C} H^{1}(\operatorname{Gal}(B / F), \mathcal{E}(B))=0 .
$$

This completes the proof of the theorem for $F / K$ finite.

We now consider the case when $F / K$ is an infinite extension. Let $L$ be any subextension of $F / K$ such that $L / K$ is finite. Consider the inflationrestriction exact sequence

$$
\begin{aligned}
& H^{1}(\operatorname{Gal}(F / L), \mathcal{E}(F)) \rightarrow H^{1}\left(\operatorname{Gal}\left(K_{\infty} / L\right), \mathcal{E}\left(K_{\infty}\right)\right) \\
& \quad \rightarrow H^{1}\left(\operatorname{Gal}\left(K_{\infty} / F\right), \mathcal{E}\left(K_{\infty}\right)\right)^{\operatorname{Gal}(F / L)} \rightarrow H^{2}(\operatorname{Gal}(F / L), \mathcal{E}(F)),
\end{aligned}
$$

which implies, after taking direct limits,

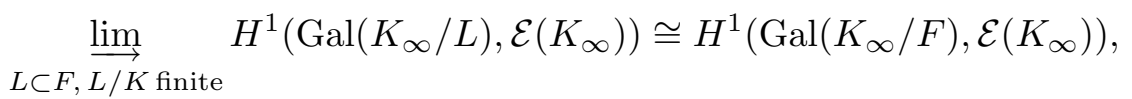

since

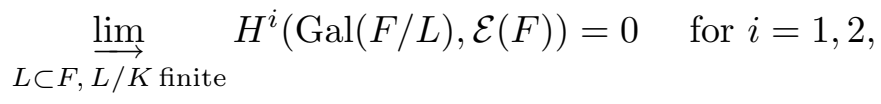

and

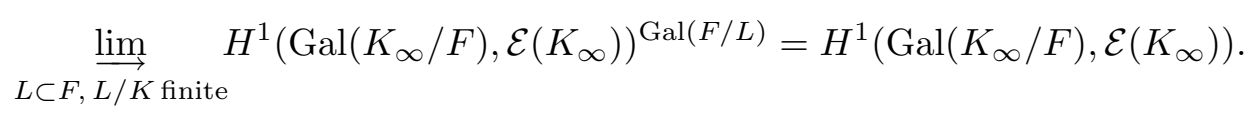

Now we have $I_{T(K)}^{d(d+4)} \mathcal{A} H^{1}\left(\operatorname{Gal}\left(K_{\infty} / F\right), \mathcal{E}\left(K_{\infty}\right)\right)=0$. This completes the proof of the theorem.

Next, we are going to show that $H^{i}\left(\operatorname{Gal}\left(K_{\infty} / F\right), \mathcal{E}\left(K_{\infty}\right)\right)$ can be annihilated by similar products for all $i \geq 2$. Since

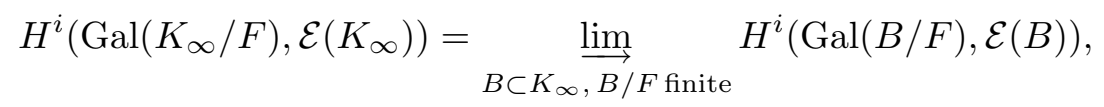

we only need to prove the following: 
TheOREM 4.7. Assume that the Iwasawa- $\mu$-conjecture is true for $K$. Also assume that for any field $F$ between $K$ and $K_{\infty}$ such that $F$ is finite over $K$, Leopoldt's conjecture holds for $F$. Let $B$ be a finite extension of $F$ contained in $K_{\infty}$. For any integer $i \geq 1$, there exists a positive integer $n$ and an ideal $\mathcal{A}$ of height at least two in $\Lambda$, both independent of $F$ and $B$, such that

$$
I_{T(K)}^{n} \mathcal{A} H^{i}(\operatorname{Gal}(B / F), \mathcal{E}(B))=0 .
$$

Pr o of. Since $K_{\infty} / K$ is a $\mathbb{Z}_{p}^{d}$-extension, $\operatorname{Gal}(B / F)$ is an abelian group which is a product of $w$ finite cyclic groups, where $w$ is an integer between 1 and $d$. We use induction on $i$.

If $i=1$, the theorem is true because of Theorem 4.6 above. Suppose it is true up to some $i \geq 1$; we need to show that it is also true for $i+1$.

If $w=1$, then $B / F$ is cyclic. This means

$$
H^{i+1}(\operatorname{Gal}(B / F), \mathcal{E}(B))=H^{1}(\operatorname{Gal}(B / F), \mathcal{E}(B))
$$

when $i$ is even, and

$$
H^{i+1}(\operatorname{Gal}(B / F), \mathcal{E}(B))=\left(\mathcal{E}(F) / \mathcal{E}(F)^{\text {univ }}\right) \otimes \mathbb{Z}_{p}
$$

when $i$ is odd. This and Theorems 4.2 and 4.6 imply that the conclusion is true in this case. Suppose that the conclusion of the theorem is true up to some $w \geq 1$. We need to show that it is also true for $w+1$.

Let $C$ be an extension of $F$ in $B$ such that $\operatorname{Gal}(B / C)$ is a product of $w$ finite cyclic groups and that $\operatorname{Gal}(C / F)$ is cyclic. Then by Section 4 of Chapter 2 in [10], we have the following Hochschild-Serre spectral sequence:

$$
H^{p}\left(\operatorname{Gal}(C / F), H^{q}(\operatorname{Gal}(B / C), \mathcal{E}(B))\right) \Rightarrow_{p} H^{*}(\operatorname{Gal}(B / F), \mathcal{E}(B)) .
$$

Using the notation in the same section of [10], we let

$$
E_{2}^{p, q}=H^{p}\left(\operatorname{Gal}(C / F), H^{q}(\operatorname{Gal}(B / C), \mathcal{E}(B))\right) .
$$

Here $p, q$ are nonnegative integers.

Since the conclusion of the theorem is true for $H^{q}(\operatorname{Gal}(B / C), \mathcal{E}(B))$ for any integer $q$ between 1 and $i+1$, there exists a positive integer $m$ and an ideal $\mathcal{B}$ of height at least two in $\Lambda$, both independent of $B$ and $C$, such that $I_{T(K)}^{m} \mathcal{B}$ annihilates $H^{q}(\operatorname{Gal}(B / C), \mathcal{E}(B))$ for all integers $q$ between 1 and $i+1$. Since $H^{0}(\operatorname{Gal}(B / C), \mathcal{E}(B))=\mathcal{E}(C)$, there exists a positive integer $l$ and an ideal $\mathcal{C}$ of height at least two in $\Lambda$, both independent of $F$ and $C$, such that

$$
I_{T(K)}^{l} \mathcal{C} H^{i+1}\left(\operatorname{Gal}(C / F), H^{0}(\operatorname{Gal}(B / C), \mathcal{E}(B))\right)=0 .
$$

This implies that there exists a positive integer $k$ and an ideal $\mathcal{D}$ of height at least two in $\Lambda$, both independent of $F, B$ or $C$, such that

$$
I_{T(K)}^{k} \mathcal{D} \bigoplus_{p+q=i+1} E_{2}^{p, q}=0 .
$$


From this we get

This means

$$
I_{T(K)}^{k} \mathcal{D} \bigoplus_{p+q=i+1} E_{\infty}^{p, q}=0
$$

$$
I_{T(K)}^{k(i+1)} \mathcal{D}^{(i+1)} H^{i+1}(\operatorname{Gal}(B / F), \mathcal{E}(B))=0 .
$$

Now we can conclude that there exists a positive integer $n$ and an ideal $\mathcal{A}$ of height at least two in $\Lambda$, both independent of $F$ and $B$, such that $I_{T(K)}^{n} \mathcal{A} H^{i+1}(\operatorname{Gal}(B / F), \mathcal{E}(B))=0$.

Acknowledgements. I would like to thank my advisor Karl Rubin for introducing the problem to me. I would also like to thank Professor Warren Sinnott and Professor Karl Rubin for helpful discussions.

\section{References}

[1] J. W. S. Cassels and A. Fröhlich (eds.), Algebraic Number Theory, Academic Press, 1967.

[2] R. Greenberg, The Iwasawa invariants of $\Gamma$-extensions of a fixed number field, Amer. J. Math. 95 (1973), 204-214.

[3] - On the structure of certain Galois groups, Invent. Math. 47 (1978), 85-99.

[4] K. Iw as awa, On $\mathbf{Z}_{l}$-extensions of algebraic number fields, Ann. of Math. 98 (1973), 246-326.

[5] - On cohomology groups of units for $\mathbf{Z}_{p}$-extensions, Amer. J. Math. 105 (1983), 189-200.

[6] S. Lang, Cyclotomic Fields, I and II, Springer, 1990.

[7] H. Matsumura, Commutative Algebra, Math. Lecture Note Ser. 56, Benjamin/ Cummings, 1980.

[8] P. Monsky, On p-adic power series, Math. Ann. 255 (1981), 217-227.

[9] K. Rubin, The "main conjecture" of Iwasawa theory for imaginary quadratic fields, Invent. Math. 103 (1991), 25-68.

[10] S. Shatz, Profinite Groups, Arithmetic, and Geometry, Princeton Univ. Press, 1972 .

[11] L. Washington, Introduction to Cyclotomic Fields, Springer, 1982.

[12] J.-P. Wintenberger, Structure galoisienne de limites projectives d'unités locales, Compositio Math. 42 (1981), 89-103.

Department of Mathematics

The Ohio State University

231 West 18th Avenue

Columbus, Ohio 43210

U.S.A.

E-mail: mingxu@math.ohio-state.edu

Received on 15.7.1997

and in revised form on 5.5.1998 